\title{
Emergence of Chikungunya Virus in Pakistan: What should be done?
}

\author{
Abbas Ali Hussain, Rabia Sarwar
}

\section{BACKGROUND:}

Since 10 years, Chikungunya - a virus spread through Aedes spp mosquitoes - has reemerged in Africa, southern and southeastern Asia, and the Indian Ocean Islands. ${ }^{1}$ In SouthEast Asia, epidemics have been documented in India, Malaysia, Sri Lanka, Myanmar, Thailand, Indonesia, the Philippines, Cambodia, Vietnam, Hong Kong, and Pakistan. ${ }^{2}$ In September 2016, despite the warning of National Institute of Health (NIH) about the possible danger of chikungunya after its outbreak in India, Pakistan suffered its initial chikungunya outbreak in Karachi, megacity of the province Sindh infecting over 3000 people. ${ }^{3}$ The very first case of Chikungunya outbreak in Pakistan was reported at a government facility in Malir, Karachi. ${ }^{4}$ According to recent studies conducted by WHO, Pakistan shares the highest burden of major vector-borne diseases such as chikungunya. ${ }^{5}$

Chikungunya virus (CHIKV) is an alpha virus belonging to family togaviridea. It exists in three genotypes, the Asian, West African and East Central South African that are responsible for outbreaks in the respective areas. Chikungunya fever is spread by the bite of mosquitoes of the genus Aedes, Aedes aegypti being the principal vector. ${ }^{2,6,7}$ A. aegypti mainly multiplies in fresh water storages such as desert coolers, water-tanks etc and around human habituation (pots, bins, cans etc) in urban and semi urban environments. The virus is postulated to exist in a human-mosquito-human circuit, and the bite of the female mosquito accounts to infection because it requires blood meal for the formation of eggs. ${ }^{2}$

\section{INTRODUCTION:}

Chikungunya fever is a disease of debilitating nature associated with a variety of symptoms. Since the Indian Ocean outbreak, information related to clinical picture of patients infected with CHIKV has increased substantially. ${ }^{7}$ According to some studies, CHIKV fever affects all age groups and both sexes equally. ${ }^{2,8}$ However males and individuals with blood Rhesus-positive were found more sensitive to get the disease in a single study. ${ }^{7}$ The abrupt onset of high grade fever with rash, arthralgia in small joints

Abbas Ali Hussain
Final Year Student MBBS
Jinnah Sindh Medical University, Karachi
Email: abbasalihusain7@gmail.com
Rabia Sarwar
Final Year Student MBBS
Jinnah Sindh Medical University, Karachi
Received: 19-Jan-2020
Accepted: 06-Apr-2020

of hands and toes, back pain and headache are the characteristic clinical signs of CHIKV fever., ${ }^{1,2,6,7,9,10}$ The disease is now described in two clinical stages: Acute stage and the late stage of illness, with long-lasting arthropathy.

The initial 10 days after the start of the disease is characterized as the acute stage of illness. ${ }^{6}$ The most usual symptoms during this stage are sudden high grade fever which responds poorly to anti-pyretics, myalgias, headache, fatigue and polyarthralgias. ${ }^{1,2,6,7}$ Joint pain associated with the fever is found in majority of the cases and is mostly polyarticular, bilateral and symmetrical involving mainly small peripheral joints (hands, wrist, and ankles) and some large joints including knee and shoulder. ${ }^{2.6 .7}$ The chronic stage is characterized by unpredicted relapses and persistence of symptoms. ${ }^{2,7}$ The affected patients may experience prolonged rheumatism with stiffness which can cause serious discomfort in walking and handling objects resulting in deterioration in the quality of life..$^{2,6,7}$ The disease may also cause neurologic, ocular or hemorrhagic symptoms atypically. ${ }^{2,6-8}$

Laboratory diagnosis is essential for a physician to diagnose Chikungunya fever and to distinguish it from diseases with similar clinical patterns like dengue virus infection. For this purpose samples collected early i.e. within a week after the infection are useful for virus detection and isolation due to high levels of viremia. ${ }^{11}$ RT-PCR and real-time loop-mediated isothermal amplification (RT-LAMP) seems to be useful in early detection of viral DNA., ${ }^{2,11}$ Viral culture by using vero cells, mosquitos or mouse models may take a week to grow and requires a laboratory with biosafety level 3 facilities, yet it remains the gold standard test for diagnosing Chikungunya fever. Serological tests for the detection of specific anti-CHIKV IgM and IgG are more frequently used. Anti-CHIKV IgM is detectable after 3-5 days of infection and remains elevated for 3-6 months. Anti-CHIKV IgG is detectable after 14 days of infection and may persist in the blood for years. Techniques like ELISA, immunofluorescence, complement binding and haemagglutination offers limited specificity and sensitivity but can be used as alternative tools for detection.

Emergence and reemergence of infectious diseases is closely associated with climatic changes, Mosquito-borne diseases are among the most sensitive to it. ${ }^{12}$ Pakistan, along with many other countries in Asia is undergoing a serious climate change which has nurtured a variety of arboviral illnesses including chikungunya fever ${ }^{3}$ In addition, wretched sanitary conditions, mountains of filth, open gutters and pools of stagnant water have contributed greatly to the outbreak. ${ }^{3}$ Each individual in Pakistan generates around $0.612 \mathrm{~kg}$ of waste per day which is increasing at a rate of $2.4 \%$. It is estimated that Karachi alone produces over 20,000 tons of 
waste per day, major share of which doesn't reach the junk sites. Moreover, overflowing gutters and stagnant water also provides a perfect habitat for arthropod vectors to grow.

\section{RECOMMENDATIONS:}

Currently, no authorized vaccine is commercially available for chikungunya fever however several vaccines have been tested with variable success rate. ${ }^{6-9}$ Moreover, treatment for chikungunya fever is highly symptomatic which includes the use of analgesics and antipyretics. ${ }^{2,6-9,11}$ Adequate oral rehydration and NSAIDs are the pillars of the treatment. For a country like Pakistan with debilitating sanitary conditions, Vector control and public awareness is crucial for the prevention of CHIKV., ${ }^{2,6,8,11}$ Public awareness should be encouraged via campaigns and seminars to eradicate CHIKV transmission. Different campaigns were conducted since the outbreak however these only educated the literate population of the community while the underprivileged people remained untaught. Educating the underprivileged will have a pivotal role in controlling the virus as it constitutes the majority in Pakistan. Limiting practice of covering only lower half of the body in hot weather and the use of mosquito repellents, bed nets and wearing long sleeves is highly recommended.

Efficient government and health ministry also plays a significant role in preventing outbreaks in a state. Despite the warnings issued by the National Institute of Health (NIH) after the outbreak in India, no precautionary measures were taken by the Pakistani Government. Airports and railway stations remained unchecked, sanitary conditions were not optimized and no productive strategies were implemented in the hospitals. ${ }^{3}$ Prevention of emerging infectious diseases are the responsibility of national governments and this cannot be delegated to any other agency. Strong political determination, educated ministers and adequate funding for preventive program management can help curtail the infection.

Early detection and accurate diagnosis is vital in controlling an infection. ${ }^{9}$ Unfortunately, due to deficiency of proper diagnosing resources in Pakistan, samples were sent to United States for detection. ${ }^{3}$ This diagnostic delay can lead to use of drugs which are contraindicated in chikungunya fever like aspirin which is an easily available over the counter drug in Pakistan and is generally self-prescribed for body aches (one of the symptoms of chikungunya fever). ${ }^{6,11}$ This can potentially increase in the misery of the patient therefore appropriate detection is important to prevent devastating outcomes of the disease. Overall, preventing an emerging infectious disease is a collaborative effort and a well-educated community with potent public health machinery can help a state to establish solid grounds in terms of health.

\section{REFERENCES:}

1. Burt FJ, Rolph MS, Rulli NE, Mahalingam S, Heise MT. Chikungunya: a re-emerging virus. The Lancet. 2012; 379 (9816):662-71

2. Mohan A, Kiran DHN, Manohar IC, Kumar DP. Epidemiology, clinical manifestations, and diagnosis of chikungunya fever: lessons learned from the re-emerging epidemic. Indian Journal of Dermatology. 2010;55(1):54-63.

3. Mallhi TH, Khan YH, Khan AH, Tanveer N, Qadir MI. First chikungunya outbreak in Pakistan: a trail of viral attacks. New Microbes and New Infections. 2017;19:13-4.

4. Kazi M. Brace yourself for new mosquito-borne disease: The Express Tribune; 2016. Available from: https://tribune. com.pk/story/1267248/alarm-bells-brace-new-mosquito-bornedisease/.

5. Malaria and other vector-borne diseases: WHO EMRO; 2015. Available from: http://www.emro.who. int/pak/programmes/ roll-back-malaria.html.

6. Simon F, Javelle E, Oliver M, Leparc-Goffart I, Marimoutou C. Chikungunya Virus Infection. Current Infectious Disease Reports. 2011;13(3):218.

7. Thiberville S-D, Moyen N, Dupuis-Maguiraga L, Nougairede A, Gould EA, Roques P, et al. Chikungunya fever: Epidemiology, clinical syndrome, pathogenesis and therapy. Antiviral Research. 2013;99(3):345-70.

8. Her Z, Kam Y-W, T.P. Lin R, Ng L. Chikungunya: a bending reality2009. 1165-76 p.

9. Sudeep AB, Parashar D. Chikungunya: an overview. Journal of Biosciences. 2008;33(4):443.

10. Wahid B, Ali A, Rafique S, Idrees M. Global expansion of chikungunya virus: mapping the 64-year history. International Journal of Infectious Diseases. 2017;58:69-76.

11. Ali I, Dasti JI. Chikungunya virus; an emerging arbovirus in Pakistan. JPMA The Journal of the Pakistan Medical Association. 2018;68(2):252-7.

12. Patz JA, Epstein PR, Burke TA, Balbus JM. Global climate change and emerging infectious diseases. JAMA. 1996;275(3):217-23. 